\title{
Self-focusing and self-trapping of optical beams upon photopolymerization
}

\author{
Anthony S. Kewitsch* and Amnon Yariv \\ Department of Applied Physics, California Institute of Technology, 128-95, Pasadena, California 91125
}

Received October 2, 1995

\begin{abstract}
We demonstrate theoretically and experimentally that optical beams are self-focused and self-trapped upon initiating photopolymerization. This unique nonlinear optical phenomenon is dependent on the optical exposure and produces permanent index-of-refraction changes larger than 0.04 . The resulting nonlinear wave equation is shown to be nonlocal in time and displays self-trapped solutions only for sufficiently low average optical intensities. (c) 1996 Optical Society of America
\end{abstract}

In this Letter we propose and demonstrate a new mechanism for self-focusing and self-trapping based on photopolymerization. Photopolymerization produces a permanent index change that is a function of absorbed optical energy rather than of intensity and is larger than the Kerr index change by orders of magnitude. Although Kerr solitons require an intensity of megawatts per square centimeter, ${ }^{1}$ we show that light beams can be self-trapped in photopolymers only for sufficiently low average intensities. The response time of the Kerr nonlinearity is on the order of femtoseconds, and the nonlinear optical response time of photopolymerization increases from milliseconds to minutes as the reaction proceeds. Like the Kerr effect, photopolymerization is a local phenomenon in space, for the index change at any location depends only on the light intensity at that same location. However, the index change is nonlocal in time, for it depends on the history of the optical electric field at all earlier times. We show that this leads to a fundamentally new form of the nonlinear wave equation that displays transient, self-guided solutions.

The propagation of light in a photopolymer is described by the nonlinear wave equation for the optical electric field. The nonlinearity arises from the dependence of the index of refraction and absorption on optical exposure. The index of refraction of the monomer during cross-linking is measured on an Abbe refractometer at $589.3 \mathrm{~nm}$. For a diacrylate and triacrylate photopolymer, the maximum index changes are 0.043 and 0.028 , respectively. Figure 1 illustrates the measured evolution of the index of refraction of the diacrylate photopolymer. The index variation with exposure displays three distinct responses. During the first stage, for low exposures, the polymer chains are small ( $<10$ monomer units). In addition, radicals are scavenged by the highly reactive oxygen molecules absorbed by the photopolymer. The bonding of two relatively small molecules induces only a small density and index-of-refraction change, corresponding to the initial flat induction period in Fig. 1. During the second stage, large polymer molecules are formed, and cross-linking between adjacent chains draws entire polymer backbones together, resulting in a large index change proportional to the density change. Unlike the Kerr effect, the index response is delayed by $0.01-$
$1 \mathrm{~s}$ relative to the illumination. The third stage exhibits saturation of the index change as the polymerization reaction approaches completion. At this final stage the diffusion constant of monomer radicals responsible for chain building has decreased dramatically to a value as small as $10^{-10} \mathrm{~cm}^{2} \mathrm{~s}^{-1}$ (Ref. 2) so that seconds to minutes are required for the index change to develop. The largest index changes within the polymer are expected to be less than or equal to $0.15,{ }^{3}$ well in excess of traditional nonlinear optical phenomena such as the Kerr and photorefractive effects, which display index changes of less than $10^{-3}$.

Experimental results such as Fig. 1 for a wide range of acrylate photopolymers indicate that the index response to an optical field amplitude $E$ is of the form

$\Delta n^{\prime}(x, y, z, t)=\Delta n_{o}^{\prime}\left\{1-\exp \left[-\frac{1}{U_{o}} \int_{0}^{t-\tau}\left|E\left(t^{\prime}\right)\right|^{2} \mathrm{~d} t^{\prime}\right]\right\}$,

where $\tau$ is the monomer radical lifetime. The expression for absorption photobleaching is approximately

$$
\Delta n^{\prime \prime}(x, y, z, t)=\Delta n_{o}^{\prime \prime} \exp \left[-\frac{1}{U_{o}} \int_{0}^{t}\left|E\left(t^{\prime}\right)\right|^{2} \mathrm{~d} t^{\prime}\right] .
$$

$U_{o}$ is the critical exposure needed to induce polymerization. Note that the real part of the index response

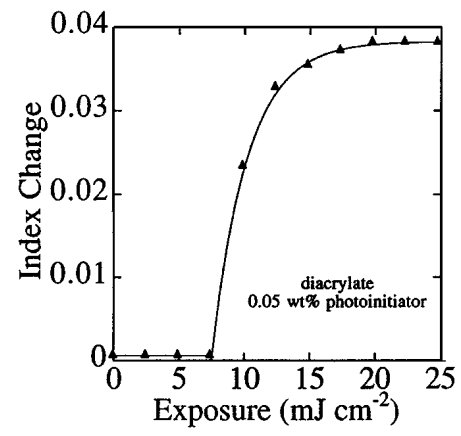

Fig. 1. Measured change in index of refraction (at $589.3 \mathrm{~nm}$ ) of the diacrylate photopolymer under UV exposure at $325 \mathrm{~nm}$. The fitted curve is given by Eq. (2) with $U_{o}$ (in energy units) equal to $2.68 \mathrm{~mJ} \mathrm{~cm}^{-2}$. 
lags behind the application of the optical field by a time delay $\tau$. This index change develops once the photogenerated radical diffuses to an appropriate monomer unit and induces cross-linking, rather than immediately after the generation of radicals. However, the absorption change associated with photoinitiator photocleavage occurs within several femtoseconds. One can reduce or even eliminate the induction period apparent for small exposures in Fig. 1 by purging the monomer of oxygen radicals. The experimental data then display good agreement with Eq. (1).

Several qualitative predictions regarding the evolution of the light-induced waveguide can be deduced from Fig. 1. At early times, the induction period and the delay in the material response impede selftrapping. The induction period causes exposure thresholding. Polymerization is initiated first in regions of intense illumination, so the lateral dimension of the waveguide is smaller than that of the optical beam. The lenslike index profile focuses primarily the central part of the beam. At later times the index increases linearly with exposure and the index distribution becomes a faithful replica of the transverse intensity profile. Self-focusing is strong, and the waveguide extends through the medium. For large exposure the index change saturates at the center of the waveguide and self-focusing is diminished. At this stage the waveguide becomes essentially a step-index fiber. Of course, for very large exposures the entire liquid volume will polymerize because the tails of the optical field extend beyond the core and into the uncured photopolymer. Although not treated by Eqs. (1) and (2), the scatter of light and the diffusion of free radicals beyond the illuminated region further contribute to the increase in waveguide diameter and the termination of guiding.

The guidance condition for an optical waveguide depends on the index change between the solid polymer core and the liquid polymer cladding. For a typical index change of 0.04 , the smallest stable diameter of the self-trapped beam is expected to be $0.6 \mu \mathrm{m}$. An additional and unique condition for self-trapping in photopolymers is that the average optical intensity must be below an approximate threshold value given by $I_{\text {ave }}<U_{o} / \tau$, where $U_{o}$ is the critical exposure needed to cure the photopolymer and $\tau$ is the monomer radical lifetime. For a typical photopolymer composition (photoinitiator concentration $0.005 \mathrm{wt} . \%, U_{o} \approx 1 \mathrm{~J} \mathrm{~cm}^{-2}$, $\tau \approx 100 \mathrm{~ms}$ ), the average intensity must be less than $10 \mathrm{~W} \mathrm{~cm}^{-2}$. The optical nonlinearity is unique in that it occurs only for low average optical intensity. Intuitively, if intense illumination were used, a large number of radicals would be produced in a time that is short compared with the radical lifetime $\tau$. The photopolymer would then cure completely in the illuminated region, before the optical beam experienced an index change.

Analytical solutions to the nonlinear wave equation with an index-of-refraction response given by Eqs. (1) and (2) are obtained only for overly simplistic approximations. We therefore analyze the propagation of the optical wave through the photopolymer numerically by solving the complete nonlinear wave equation. The intensity profile is computed by the beam-propagation method, ${ }^{4,5}$ incorporating the transparent boundary condition ${ }^{6}$ and the measured index evolution. Typical photopolymer compositions used in these experiments have low optical absorption associated with the photoinitiator, so photobleaching described by Eq. (2) can be ignored.

Two-dimensional numerical simulations exhibit selftrapped solutions to the nonlinear wave equation for low average optical intensities. Figure 2 illustrates the electric field amplitude of the self-trapped Gauss-

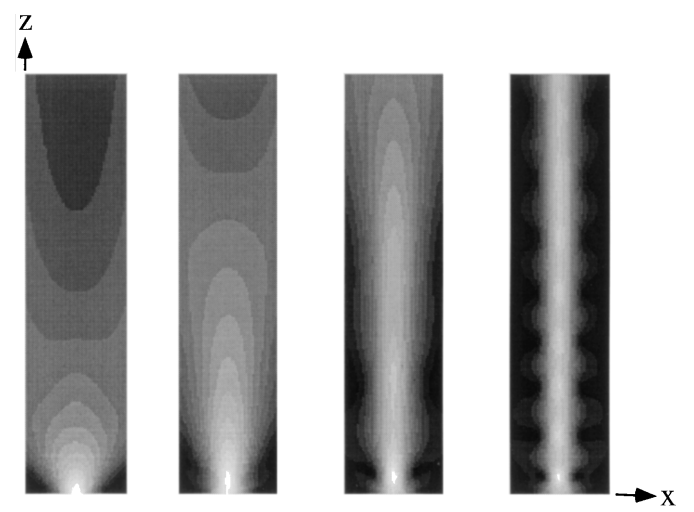

Fig. 2. Numerical simulations of beam propagation in photopolymer in time steps equal to $5 t_{c}$. Left-most simulation, $t=0$; right-most simulation, $t=15 t_{c}$. The vertical scale is $10^{3}$ wavelengths; the horizontal scale is 10 wavelengths.

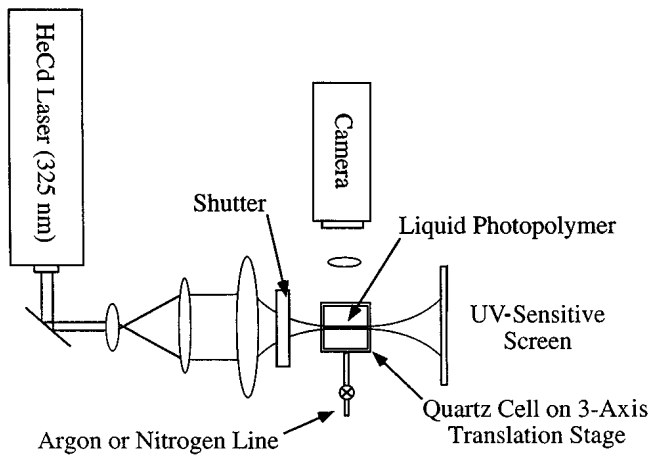

Fig. 3. Experimental setup for self-focusing and selftrapping of optical beams. The input power is approximately $5 \mathrm{~mW}$ at $325 \mathrm{~nm}$.
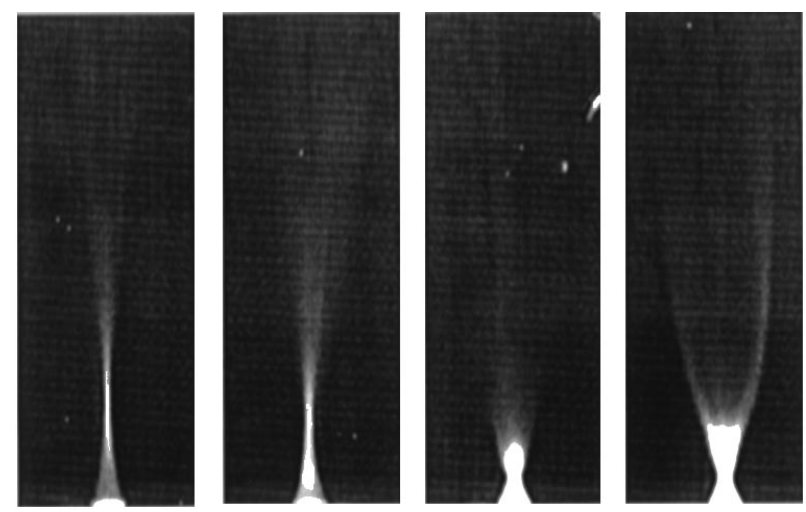

Fig. 4. Experimental demonstration of self-focusing upon photopolymerization. Left-most simulation, photopolymer at $t=0$; right-most simulation, photopolymer at $t=$ $30 \mathrm{~s}$. The horizontal scale is $300 \mu \mathrm{m}$; the vertical scale is $1 \mathrm{~mm}$. 


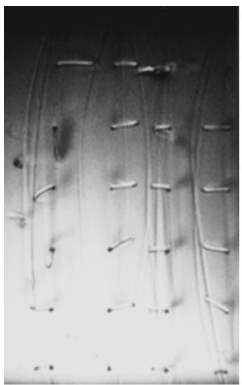

(a)

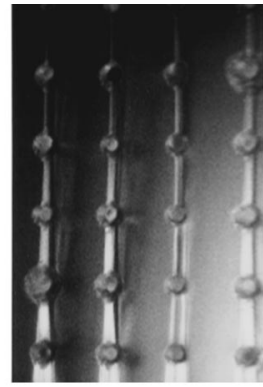

(b)

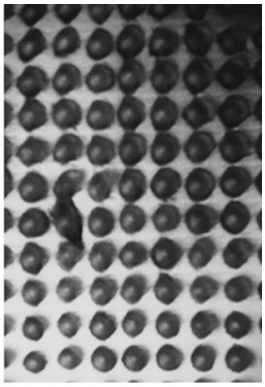

(c)
Fig. 5. (a) Series of fibers grown by self-trapping of the UV beam; the nominal diameter is $10 \mu \mathrm{m}$. (b) Threedimensional fiber lattice with $50-\mu \mathrm{m}$-diameter filaments of 5-mm length, fabricated within a photopolymer-filled cuvette. (c) Array of fibers of $200-\mu \mathrm{m}$ diameter and 10-mm length.

ian beam in a photopolymer liquid. The waist of the beam is located at the input surface of the photopolymer. The beam width at the input is equal to two wavelengths, and the propagation distance is 500 wavelengths. Note that the horizontal scale is magnified by a factor of 10 compared with the vertical scale, to improve the transverse resolution. The left-most simulation illustrates the evolution of the beam through the homogeneous photopolymer before any index changes are induced. The time steps from the left to the right are in units of $5 t_{c} . \quad t_{c}$ is a parameter chosen to equal the time needed to attain the critical exposure $U_{o}$ at the location of maximum intensity of the optical beam. Self-trapping of the optical beam is apparent after an exposure of $15 t_{c}$. The weak oscillations in the beam diameter evident at $15 t_{c}$ are typical for selftrapped beams. ${ }^{7}$ The background absorption length is $10^{3}$ wavelengths, so self-trapping terminates in approximately this distance.

We have experimentally observed self-focusing in a liquid diacrylate monomer using the experimental setup of depicted in Fig. 3. The input beam is a $1-\mathrm{mW}, \mathrm{TEM}_{00}$ mode at $325 \mathrm{~nm}$ with a $100-\mu \mathrm{m}$ beam diameter at the entrance face. The left-most simulation in Fig. 4 illustrates the beam profile at $t=0$ inside the liquid photopolymer. Time increases from left to right. The right-most simulation in Fig. 4 is taken after approximately $30 \mathrm{~s}$ of continuous illumination. The position of the beam waist moves closer to the input plane as photopolymerization proceeds, a manifestation of self-focusing.

We observe dramatic self-trapping of optical beams over distances in excess of $3 \mathrm{~cm}$ and diameters of $10-50 \mu \mathrm{m}$ by exposing the polymer to a sequence of short exposures separated by a diffusion time. Figure 5(a) shows a series of solid polymer fibers $10 \mu \mathrm{m}$ in diameter and $3 \mathrm{~mm}$ in length inside a diacrylate photopolymer. Since the solid is denser than the liquid surroundings, the structures bend downward from the entrance face of the cuvette after several hours. The waveguides can be steered during formation by introduction of an asymmetry across the transverse intensity profile of the beam. Each fiber is exposed 30 times for $1 / 16 \mathrm{~s}$, with a delay of $1-10 \mathrm{~s}$ be- tween successive exposures to ensure that the average intensity is below the threshold value given above. An array of fiberlike structures can be fabricated to produce a three-dimensional microlattice [Fig. 5(b)]. The diameter of each solid filament is $50 \mu \mathrm{m}$, spaced by approximately $500 \mu \mathrm{m}$. The beam is guided over a distance greater than $10 \mathrm{~mm}$. Regular arrays of posts of $200-\mu \mathrm{m}$ diameter and $10-\mathrm{mm}$ length have also been fabricated in an epoxy-triacrylate photopolymer, as illustrated in Fig. 5(c). Each post is formed by a single $1 / 8$-s exposure, so the average intensity is too large to exhibit trapping. Hence, the diameters of the waveguides are significantly larger.

The diameters of the fibers in Figs. 5(a) and 5(b) remain constant well beyond the confocal parameter of $122 \mu \mathrm{m}$ for a Gaussian input beam with a $3-\mu \mathrm{m}$ waist. This is a signature of self-trapping. The diameter of the guided beam is highly dependent on the composition of the photopolymer, its oxygen content, and the optical intensity. The self-trapped beam is found to survive over a distance approximately equal to the absorption length. These findings show good qualitative agreement with the numerical simulations. Note that the oscillations in waveguide diameter predicted numerically are of sufficiently small amplitude ( $\sim 1$ wavelength) that we do not expect to resolve them experimentally.

In summary, we have demonstrated theoretically and experimentally that optical beams are self-focused and self-trapped upon photopolymerization. The resulting nonlinear wave equation is unique in that it is nonlocal in time and displays transient yet pronounced self-trapped solutions. Another unique feature of this nonlinear optical effect is its slow time response. This phenomenon belongs to an entirely new class of optical nonlinearity observed only at sufficiently low average optical intensity. We believe that this nonlinear optical effect may have fundamental technological applications to microfabrication, in which photopolymers are ubiquitous as photoresists for photolithography and micromachining.

This work was supported by the Advanced Research Project Agency's nonlinear optics (DSO) and lithography (MTO) programs and U.S. Army Research Office contract DAAH04-95-C-0063.

*Permanent address, Arroyo Optics, Inc., 1646 17th Street, Santa Monica, California 90404.

\section{References}

1. R. Y. Chiao, E. Garmire, and C. H. Townes, Phys. Rev. Lett. 13, 479 (1964).

2. G. Odian, Principles of Polymerization (Wiley, New York, 1981).

3. W. J. Tomlinson and E. A. Chandross, in Advances in Photochemistry, J. J. N. Pitts, G. S. Hammond, and K. Gollnick, eds. (Wiley, New York, 1985), Vol. 12, pp. 201-281

4. Y. Chung and N. Dagli, IEEE J. Quantum Electron. 26, 1335 (1990).

5. L. Sun and G. L. Yip, Opt. Lett. 18, 1229 (1993).

6. G. R. Hadley, IEEE J. Quantum Electron. 28, 363 (1992).

7. J. H. Marburger and E. Dawes, Phys. Rev. Lett. 21, 556 (1968). 